\title{
ON A NONLOCAL CAUCHY PROBLEM FOR DIFFERENTIAL INCLUSIONS
}

\author{
E. GATSORI, S. K. NTOUYAS, AND Y. G. SFICAS
}

Received 3 September 2002

We establish sufficient conditions for the existence of solutions for semilinear differential inclusions, with nonlocal conditions. We rely on a fixed-point theorem for contraction multivalued maps due to Covitz and Nadler and on the Schaefer's fixed-point theorem combined with lower semicontinuous multivalued operators with decomposable values.

\section{Introduction}

In this paper, we are concerned with proving the existence of solutions of differential inclusions, with nonlocal initial conditions. More precisely, in Section 2, we consider the following differential inclusion, with nonlocal initial conditions:

$$
\begin{gathered}
y^{\prime} \in F(t, y), \quad t \in J=[0, b], \\
y(0)+\sum_{k=1}^{p} c_{k} y\left(t_{k}\right)=y_{0},
\end{gathered}
$$

where $F: J \times \mathbb{R}^{n} \rightarrow \mathscr{P}\left(\mathbb{R}^{n}\right)$ is a multivalued map, $\mathscr{P}\left(\mathbb{R}^{n}\right)$ is the family of all subsets of $\mathbb{R}^{n}$, $y_{0} \in \mathbb{R}^{n}$, and $0 \leq t_{1}<t_{2}<\cdots<t_{p} \leq b, p \in \mathbb{N}, c_{k} \neq 0, k=1,2, \ldots, p$.

The single-valued case of problem (1.1) was studied by Byszewski [5], in which a new definition of mild solution was introduced. In the same paper, it was remarked that the constants $c_{k}, k=1, \ldots, p$, from condition (1.1b) can satisfy the inequalities $\left|c_{k}\right| \geq 1, k=$ $1, \ldots, p$. As pointed out by Byszewski [4], the study of initial value problems with nonlocal conditions is of significance since they have applications in problems in physics and other areas of applied mathematics.

The initial value problem (1.1) was studied by Benchohra and Ntouyas [1] in the semilinear case where the right-hand side is assumed to be convex-valued. Here, we drop this restriction and consider problem (1.1) with a nonconvex-valued right-hand side. By using the fixed-point theorem for contraction multivalued maps due to Covitz and Nadler [7] and the Schaefer's theorem combined with a selection theorem of Bressan 
and Colombo for lower semicontinuous (1.s.c.) multivalued operators with decomposable values, existence results are proposed for problem (1.1).

In this section, we introduce notations, definitions, and preliminary facts from multivalued analysis, which are used throughout this paper.

We denote by $\mathscr{P}(E)$ the set of all subsets of $E$ normed by $\|\cdot\|_{\mathscr{P}}$ and by $C\left(J, \mathbb{R}^{n}\right)$ the Banach space of all continuous functions from $J$ into $\mathbb{R}^{n}$ with the norm

$$
\|y\|_{\infty}=\sup \{|y(t)|: t \in J\}
$$

Also, $L^{1}\left(J, \mathbb{R}^{n}\right)$ denotes the Banach space of measurable functions $y: J \rightarrow \mathbb{R}^{n}$ which are Lebesgue integrable and normed by

$$
\|y\|_{L^{1}}=\int_{0}^{b}|y(t)| d t
$$

Let $A$ be a subset of $J \times \mathbb{R}^{n}$. The set $A$ is $\mathscr{L} \otimes \mathscr{B}$ measurable if $A$ belongs to the $\sigma$ algebra generated by all sets of the form $N \times D$, where $N$ is Lebesgue measurable in $J$ and $D$ is Borel measurable in $\mathbb{R}^{n}$. A subset $B$ of $L^{1}\left(J, \mathbb{R}^{n}\right)$ is decomposable if, for all $u, v \in B$ and $N \subset J$ measurable, the function $u \chi_{N}+v \chi_{J-N} \in B$, where $\chi$ denotes the characteristic function.

Let $E$ be a Banach space, $X$ a nonempty closed subset of $E$, and $G: X \rightarrow \mathscr{P}(E)$ a multivalued operator with nonempty closed values. The operator $G$ is l.s.c. if the set $\{x \in X: G(x) \cap C \neq \varnothing\}$ is open for any open set $C$ in $E$. The operator $G$ has a fixed point if there is $x \in X$ such that $x \in G(x)$. For more details on multivalued maps, we refer to Deimling [8], Górniewicz [10], Hu and Papageorgiou [11], and Tolstonogov [13].

Definition 1.1. Let $Y$ be a separable metric space and let $N: Y \rightarrow \mathscr{P}\left(L^{1}\left(J, \mathbb{R}^{n}\right)\right)$ be a multivalued operator. The operator $N$ has property (BC) if

(1) $N$ is l.s.c.;

(2) $N$ has nonempty closed and decomposable values.

Let $F: J \times \mathbb{R}^{n} \rightarrow \mathscr{P}\left(\mathbb{R}^{n}\right)$ be a multivalued map with nonempty compact values. Assign to $F$ the multivalued operator

$$
\mathscr{F}: C\left(J, \mathbb{R}^{n}\right) \longrightarrow \mathscr{P}\left(L^{1}\left(J, \mathbb{R}^{n}\right)\right)
$$

by letting

$$
\mathscr{F}(y)=\left\{w \in L^{1}\left(J, \mathbb{R}^{n}\right): w(t) \in F(t, y(t)) \text { for a.e. } t \in J\right\}
$$

The operator $\mathscr{F}$ is called the Niemytzki operator associated with $F$.

Definition 1.2. Let $F: J \times \mathbb{R}^{n} \rightarrow \mathscr{P}\left(\mathbb{R}^{n}\right)$ be a multivalued function with nonempty compact values. The multivalued map $F$ is of l.s.c. type if its associated Niemytzki operator $\mathscr{F}^{\circ}$ is l.s.c. and has nonempty closed and decomposable values.

Next, we state a selection theorem due to Bressan and Colombo [3]. 
Theorem 1.3 (see [3]). Let $Y$ be a separable metric space and let $N: Y \rightarrow \mathscr{P}\left(L^{1}\left(J, \mathbb{R}^{n}\right)\right)$ be a multivalued operator which has property $(B C)$. Then $N$ has a continuous selection, that is, there exists a (single-valued) continuous function $g: Y \rightarrow L^{1}\left(J, \mathbb{R}^{n}\right)$ such that $g(y) \in N(y)$ for every $y \in Y$.

Let $(X, d)$ be a metric space. We use the following notations:

$$
\begin{aligned}
P(X) & =\{Y \in \mathscr{P}(X): Y \neq \varnothing\}, \\
P_{c l}(X) & =\{Y \in P(X): Y \text { closed }\}, \\
P_{b}(X) & =\{Y \in P(X): Y \text { bounded }\}, \\
P_{c p}(X) & =\{Y \in P(X): Y \text { compact }\} .
\end{aligned}
$$

Consider $H_{d}: P(X) \times P(X) \rightarrow \mathbb{R}_{+} \cup\{\infty\}$ given by

$$
H_{d}(A, B)=\max \left\{\sup _{a \in A} d(a, B), \sup _{b \in B} d(A, b)\right\},
$$

where $d(A, b)=\inf _{a \in A} d(a, b)$ and $d(a, B)=\inf _{b \in B} d(a, b)$.

Then $\left(P_{b, c l}(X), H_{d}\right)$ is a metric space and $\left(P_{c l}(X), H_{d}\right)$ is a generalized metric space.

Definition 1.4. A multivalued operator $N: X \rightarrow P_{c l}(X)$ is called

(a) $\gamma$-Lipschitz if and only if there exists $\gamma>0$ such that

$$
H_{d}(N(x), N(y)) \leq \gamma d(x, y) \quad \text { for each } x, y \in X
$$

(b) a contraction if and only if it is $\gamma$-Lipschitz with $\gamma<1$.

For more details on multivalued maps and the proofs of known results cited in this section, we refer to Deimling [8], Górniewicz [10], Hu and Papageorgiou [11], and Tolstonogov [13].

In the sequel, we will use the following fixed-point theorem for contraction multivalued operators given by Covitz and Nadler [7] (see also Deimling [8, Theorem 11.1]).

Lemma 1.5. Let $(X, d)$ be a complete metric space. If $N: X \rightarrow P_{c l}(X)$ is a contraction, then fix $N \neq \varnothing$.

\section{Main results}

Definition 2.1. Assume that $\sum_{k=1}^{p} c_{k} \neq-1$. A function $y \in C\left(J, \mathbb{R}^{n}\right)$ is called a mild solution of (1.1) if there exists a function $v \in L^{1}\left(J, \mathbb{R}^{n}\right)$ such that $v(t) \in F(t, y(t))$ a.e. on $J$, and

$$
y(t)=A\left(y_{0}-\sum_{k=1}^{p} c_{k} \int_{0}^{t_{k}} v(s) d s\right)+\int_{0}^{t} v(s) d s
$$

where $A=\left(1+\sum_{k=1}^{p} c_{k}\right)^{-1}$. 
We will need the following assumptions:

(H1) $F: J \times \mathbb{R}^{n} \rightarrow P_{c p}\left(\mathbb{R}^{n}\right)$ has the property that $F(\cdot, y): J \rightarrow P_{c p}\left(\mathbb{R}^{n}\right)$ is measurable for each $y \in \mathbb{R}^{n}$

(H2) there exists $l \in L^{1}\left(J, \mathbb{R}^{+}\right)$such that

$$
\begin{gathered}
H_{d}(F(t, y), F(t, \bar{y})) \leq l(t)|y-\bar{y}| \quad \text { for almost each } t \in J, y, \bar{y} \in \mathbb{R}^{n}, \\
d(0, F(t, 0)) \leq \ell(t) \quad \text { for almost each } t \in J
\end{gathered}
$$

(H3) assume that

$$
\sum_{k=1}^{p} c_{k} \neq-1
$$

(H4) $|A| \sum_{k=1}^{p}\left|c_{k}\right| L\left(t_{k}\right)+L(b)<1$, where $L(t)=\int_{0}^{t} l(s) d s$.

Theorem 2.2. Assume that hypotheses (H1), (H2), (H3), and (H4) are satisfied. Then problem (1.1) has at least one mild solution on J.

Proof. Transform problem (1.1) into a fixed-point problem. Consider the multivalued operator $N: C\left(J, \mathbb{R}^{n}\right) \rightarrow \mathscr{P}\left(C\left(J, \mathbb{R}^{n}\right)\right)$ defined by

$$
N(y):=\left\{h \in C\left(J, \mathbb{R}^{n}\right): h(t)=A\left(y_{0}-\sum_{k=1}^{p} c_{k} \int_{0}^{t_{k}} g(s) d s\right)+\int_{0}^{t} g(s) d s: g \in S_{F, y}\right\},
$$

where

$$
S_{F, y}=\left\{g \in L^{1}\left(J, \mathbb{R}^{n}\right): g(t) \in F(t, y(t)) \text { for a.e. } t \in J\right\} .
$$

We will show that $N$ satisfies the assumptions of Lemma 1.5. The proof will be given in two steps.

Step 1. We prove that $N(y) \in P_{c l}\left(C\left(J, \mathbb{R}^{n}\right)\right)$ for each $y \in C\left(J, \mathbb{R}^{n}\right)$.

Indeed, let $\left(y_{n}\right)_{n \geq 0} \in N(y)$ such that $y_{n} \rightarrow \tilde{y}$ in $C\left(J, \mathbb{R}^{n}\right)$. Then $\tilde{y} \in C\left(J, \mathbb{R}^{n}\right)$ and there exist $g_{n} \in S_{F, y}$ such that

$$
y_{n}(t)=A\left(y_{0}-\sum_{k=1}^{p} c_{k} \int_{0}^{t_{k}} g_{n}(s) d s\right)+\int_{0}^{t} g_{n}(s) d s
$$

Using the fact that $F$ has compact values, and from $(\mathrm{H} 2)$, we may pass to a subsequence if necessary to get that $g_{n}$ converges to $g$ in $L^{1}(J, E)$ and hence $g \in S_{F, y}$. Then for each $t \in[0, b]$

$$
y_{n}(t) \longrightarrow \tilde{y}(t)=A\left(y_{0}-\sum_{k=1}^{p} c_{k} \int_{0}^{t_{k}} g(s) d s\right)+\int_{0}^{t} g(s) d s
$$

So, $\tilde{y} \in N(y)$.

Step 2. We prove that $H_{d}\left(N\left(y_{1}\right), N\left(y_{2}\right)\right) \leq \gamma\left\|y_{1}-y_{2}\right\|_{\infty}$ for each $y_{1}, y_{2} \in C\left(J, \mathbb{R}^{n}\right)$ (where $\gamma<1$ ). 
Let $y_{1}, y_{2} \in C\left(J, \mathbb{R}^{n}\right)$ and $h_{1} \in N\left(y_{1}\right)$. Then there exists $g_{1}(t) \in F\left(t, y_{1}(t)\right)$ such that

$$
h_{1}(t)=A\left(y_{0}-\sum_{k=1}^{p} c_{k} \int_{0}^{t_{k}} g_{1}(s) d s\right)+\int_{0}^{t} g_{1}(s) d s, \quad t \in J .
$$

From (H2), it follows that

$$
H_{d}\left(F\left(t, y_{1}(t)\right), F\left(t, y_{2}(t)\right)\right) \leq l(t)\left|y_{1}(t)-y_{2}(t)\right|, \quad t \in J .
$$

Hence, there is $w \in F\left(t, y_{2}(t)\right)$ such that

$$
\left|g_{1}(t)-w\right| \leq l(t)\left|y_{1}(t)-y_{2}(t)\right|, \quad t \in J .
$$

Consider $U: J \rightarrow \mathscr{P}\left(\mathbb{R}^{n}\right)$ given by

$$
U(t)=\left\{w \in \mathbb{R}^{n}:\left|g_{1}(t)-w\right| \leq l(t)\left|y_{1}(t)-y_{2}(t)\right|\right\} .
$$

Since the multivalued operator $V(t)=U(t) \cap F\left(t, y_{2}(t)\right)$ is measurable (see [6, Proposition III.4]), there exists $g_{2}(t)$ a measurable selection for $V$. So, $g_{2}(t) \in F\left(t, y_{2}(t)\right)$ and

$$
\left|g_{1}(t)-g_{2}(t)\right| \leq l(t)\left|y_{1}(t)-y_{2}(t)\right| \quad \text { for each } t \in J \text {. }
$$

We define for each $t \in J$,

$$
h_{2}(t)=A\left(y_{0}-\sum_{k=1}^{p} c_{k} \int_{0}^{t_{k}} g_{2}(s) d s\right)+\int_{0}^{t} g_{2}(s) d s, \quad t \in J .
$$

Then we have

$$
\begin{aligned}
\left|h_{1}(t)-h_{2}(t)\right| \leq & \left|A \sum_{k=1}^{p} c_{k} \int_{0}^{t_{k}}\left[g_{1}(s)-g_{2}(s)\right] d s+\int_{0}^{t}\left[g_{1}(s)-g_{2}(s)\right] d s\right| \\
\leq & |A| \sum_{k=1}^{p}\left|c_{k}\right|\left\|y_{1}-y_{2}\right\|_{\infty} \int_{0}^{t_{k}} \ell(s) d s \\
& +\left\|y_{1}-y_{2}\right\|_{\infty} \int_{0}^{t} l(s) d s \\
\leq & \left(|A| \sum_{k=1}^{p}\left|c_{k}\right| L\left(t_{k}\right)+L(b)\right)\left\|y_{1}-y_{2}\right\|_{\infty} .
\end{aligned}
$$

Then

$$
\left\|h_{1}-h_{2}\right\|_{\infty} \leq\left(|A| \sum_{k=1}^{p}\left|c_{k}\right| L\left(t_{k}\right)+L(b)\right)\left\|y_{1}-y_{2}\right\|_{\infty} .
$$

By the analogous relation obtained by interchanging the roles of $y_{1}$ and $y_{2}$, it follows that

$$
H_{d}\left(N\left(y_{1}\right), N\left(y_{2}\right)\right) \leq\left(|A| \sum_{k=1}^{p}\left|c_{k}\right| L\left(t_{k}\right)+L(b)\right)\left\|y_{1}-y_{2}\right\|_{\infty} .
$$


From (H4), we have that

$$
\gamma:=|A| \sum_{k=1}^{p}\left|c_{k}\right| L\left(t_{k}\right)+L(b)<1 .
$$

Then $N$ is a contraction, and thus, by Lemma 1.5, it has a fixed point $y$ which is a mild solution to (1.1).

Remark 2.3. Consider the Bielecki-type norm (see [2]) on $C\left(J, \mathbb{R}^{n}\right)$, defined by

$$
\|y\|_{\Re}=\max _{t \in J}\left\{|y(t)| e^{-\tau L(t)}\right\}
$$

where $L(t)=\int_{0}^{t} l(s) d s$. Since

$$
e^{-\tau L(b)}\|y\|_{\infty} \leq\|y\|_{\mathscr{B}} \leq\|y\|_{\infty},
$$

the norms $\|y\|_{\mathscr{B}}$ and $\|y\|_{\infty}$ are equivalent.

Then we can prove Step 2 of Theorem 2.2, that is, $H_{d}\left(N\left(y_{1}\right), N\left(y_{2}\right)\right) \leq \gamma\left\|y_{1}-y_{2}\right\|_{\circledast}$ for each $y_{1}, y_{2} \in C\left(J, \mathbb{R}^{n}\right)$, where

$$
\gamma=\frac{1}{\tau}\left(|A| \sum_{k=1}^{p}\left|c_{k}\right| e^{\tau L\left(t_{k}\right)}+1\right) .
$$

Indeed, we have

$$
\begin{aligned}
\left\|h_{1}-h_{2}\right\|_{\mathscr{B}}= & \max _{t \in J} e^{-\tau L(t)} \mid A \sum_{k=1}^{p} c_{k} \int_{0}^{t_{k}}\left[g_{1}(s)-g_{2}(s)\right] d s \\
& +\int_{0}^{t}\left[g_{1}(s)-g_{2}(s)\right] d s \mid \\
\leq & |A| \sum_{k=1}^{p}\left|c_{k}\right||| y_{1}-y_{2} \|_{\Re} \int_{0}^{t_{k}} \ell(s) e^{\tau L(s)} d s \\
& +|| y_{1}-y_{2}||_{\mathscr{B}} \int_{0}^{t} l(s) e^{\tau L(s)} d s \\
\leq & \left(|A| \sum_{k=1}^{p}\left|c_{k}\right| \frac{e^{\tau L\left(t_{k}\right)}}{\tau}+\frac{1-e^{-\tau L(b)}}{\tau}\right)\left\|y_{1}-y_{2}\right\|_{\Re} \\
\leq & \left(|A| \sum_{k=1}^{p}\left|c_{k}\right| \frac{e^{\tau L\left(t_{k}\right)}}{\tau}+\frac{1}{\tau}\right)\left\|y_{1}-y_{2}\right\|_{\mathscr{B}} .
\end{aligned}
$$

We can choose $\tau$ such that $\gamma<1$. In this case, (H4) must be deleted.

By the help of the Schaefer's fixed-point theorem combined with the selection theorem of Bressan and Colombo for l.s.c. maps with decomposable values, we will present an existence result for problem (1.1). Before this, we introduce the following hypotheses 
which are assumed hereafter:

(H5) $F: J \times C\left(J, \mathbb{R}^{n}\right) \rightarrow \mathscr{P}\left(\mathbb{R}^{n}\right)$ is a nonempty compact-valued multivalued map such that

(a) $(t, u) \mapsto F(t, u)$ is $\mathscr{L} \otimes \mathscr{B}$ measurable;

(b) $u \mapsto F(t, u)$ is l.s.c. for a.e. $t \in J$;

(H6) for each $r>0$, there exists a function $h_{r} \in L^{1}\left(J, \mathbb{R}^{+}\right)$such that

$$
\|F(t, u)\|_{\mathscr{P}}:=\sup \{|v|: v \in F(t, u)\} \leq h_{r}(t) \quad \text { for a.e. } t \in J, u \in \mathbb{R}^{n} \text { with }|u| \leq r .
$$

In the proof of Theorem 2.5, we will need the next auxiliary result.

Lemma 2.4 (see [9]). Let $F: J \times C\left(J, \mathbb{R}^{n}\right) \rightarrow \mathscr{P}\left(\mathbb{R}^{n}\right)$ be a multivalued map with nonempty, compact values. Assume that (H5) and (H6) hold. Then F is of l.s.c. type.

Theorem 2.5. Suppose, in addition to hypotheses (H5) and (H6), that the following also holds:

(H7) Assume that $\|F(t, y)\|_{\mathscr{P}}:=\sup \{|v|: v \in F(t, y)\} \leq p(t) \psi(|y|)$ for almost all $t \in J$ and all $y \in \mathbb{R}^{n}$, where $p \in L^{1}\left(J, \mathbb{R}_{+}\right)$and $\psi: \mathbb{R}_{+} \rightarrow(0, \infty)$ is continuous and increasing with

$$
\int^{\infty} \frac{d u}{\psi(u)}=\infty
$$

Then the initial value problem (1.1) has at least one solution on J.

Proof. By Lemma 2.4, (H5) and (H6) imply that $F$ is of 1.s.c. type. Then, from Theorem 1.3 , there exists a continuous function $f: C\left(J, \mathbb{R}^{n}\right) \rightarrow L^{1}\left(J, \mathbb{R}^{n}\right)$ such that $f(y) \in \mathscr{F}(y)$ for all $y \in C\left(J, \mathbb{R}^{n}\right)$.

We consider the problem

$$
\begin{gathered}
y^{\prime}(t)=f(y)(t), \quad t \in J, \\
y(0)+\sum_{k=1}^{p} c_{k} y\left(t_{k}\right)=y_{0} .
\end{gathered}
$$

We remark that if $y \in C\left(J, \mathbb{R}^{n}\right)$ is a solution of problem (2.24), then $y$ is a solution to problem (1.1).

Transform problem (2.24) into a fixed-point problem by considering the operator $N_{1}$ : $C\left(J, \mathbb{R}^{n}\right) \rightarrow C\left(J, \mathbb{R}^{n}\right)$ defined by

$$
N_{1}(y)(t):=A\left(y_{0}-\sum_{k=1}^{p} c_{k} \int_{0}^{t_{k}} f(y)(s) d s\right)+\int_{0}^{t} f(y)(s) d s
$$

We will show that $N_{1}$ is a compact operator. 
Step 1. The operator $N_{1}$ is continuous.

Let $\left\{y_{n}\right\}$ be a sequence such that $y_{n} \rightarrow y$ in $C\left(J, \mathbb{R}^{n}\right)$. Then

$$
\begin{aligned}
\left|N_{1}\left(y_{n}\right)(t)-N_{1}(y)(t)\right| \leq & |A| \sum_{k=1}^{p}\left|c_{k}\right| \int_{0}^{t_{k}}\left|f\left(y_{n}\right)(s)-f(y)(s)\right| d s \\
& +\int_{0}^{t}\left|f\left(y_{n}\right)(s)-f(y)(s)\right| d s \\
\leq & |A| \sum_{k=1}^{p}\left|c_{k}\right| \int_{0}^{b}\left|f\left(y_{n}\right)(s)-f(y)(s)\right| d s \\
& +\int_{0}^{b}\left|f\left(y_{n}\right)(s)-f(y)(s)\right| d s .
\end{aligned}
$$

Since the function $f$ is continuous, then

$$
\left\|N_{1}\left(y_{n}\right)-N_{1}(y)\right\|_{\infty} \longrightarrow 0 \text { as } n \longrightarrow \infty .
$$

Step 2. The operator $N_{1}$ maps bounded sets into bounded sets in $C\left(J, \mathbb{R}^{n}\right)$.

Indeed, it is enough to show that there exists a positive constant $c$ such that for each $y \in B_{q}=\left\{y \in C(J, E):\|y\|_{\infty} \leq q\right\}$, one has $\left\|N_{1}(y)\right\|_{\infty} \leq c$. By (H6), we have for each $t \in J$,

$$
\begin{aligned}
\left|N_{1}(y)(t)\right| & \leq|A|\left(\left|y_{0}\right|+\sum_{k=1}^{p}\left|c_{k}\right| \int_{0}^{t_{k}}|f(y)(s)| d s\right)+\int_{0}^{t}|f(y)(s)| d s \\
& \leq|A|\left(\left|y_{0}\right|+\sum_{k=1}^{p}\left|c_{k}\right|\left\|h_{q}\right\|_{L^{1}}\right)+\left\|h_{q}\right\|_{L^{1}\left(J, \mathbb{R}_{+}\right)} .
\end{aligned}
$$

Step 3. The operator $N_{1}$ maps bounded sets into equicontinuous sets of $C\left(J, \mathbb{R}^{n}\right)$.

Let $\tau_{1}, \tau_{2} \in J, \tau_{1}<\tau_{2}$, and $B_{q}=\left\{y \in C\left(J, \mathbb{R}^{n}\right):\|y\|_{\infty} \leq q\right\}$ a bounded set of $C(J, E)$. Thus,

$$
\left|N_{1}(y)\left(\tau_{2}\right)-N_{1}(y)\left(\tau_{1}\right)\right| \leq \int_{\tau_{1}}^{\tau_{2}} h_{q}(s) d s
$$

As $\tau_{2} \rightarrow \tau_{1}$, the right-hand side of the above inequality tends to zero.

As a consequence of Steps 1, 2, and 3, together with the Arzelá-Ascoli theorem, we can conclude that $N_{1}$ is completely continuous.

Step 4. Now, it remains to show that the set

$$
\mathscr{E}\left(N_{1}\right):=\left\{y \in C\left(J, \mathbb{R}^{n}\right): y=\lambda N_{1}(y) \text { for some } 0<\lambda<1\right\}
$$

is bounded.

Let $y \in \mathscr{E}\left(N_{1}\right)$. Then $y=\lambda N_{1}(y)$ for some $0<\lambda<1$ and

$$
y(t)=\lambda A\left(y_{0}-\sum_{k=1}^{p} c_{k} \int_{0}^{t_{k}} f(y)(s) d s\right)+\lambda \int_{0}^{t} f(y)(s) d s, \quad t \in J .
$$


This implies, by (H7), that for each $t \in J$, we have

$$
|y(t)| \leq|A|\left|y_{0}\right|+|A| \sum_{k=1}^{p}\left|c_{k}\right| \int_{0}^{t_{k}} p(t) \psi(|y(t)|) d t+\int_{0}^{t} p(s) \psi(|y(s)|) d s .
$$

We take the right-hand side of the above inequality as $v(t)$, then we have

$$
\begin{gathered}
v(0)=|A|\left|y_{0}\right|+|A| \sum_{k=1}^{p}\left|c_{k}\right| \int_{0}^{t_{k}} p(t) \psi(|y(t)|) d t, \quad|y(t)| \leq v(t), t \in J, \\
v^{\prime}(t)=p(t) \psi(|y(t)|), \quad t \in J .
\end{gathered}
$$

Using the nondecreasing character of $\psi$, we get

$$
v^{\prime}(t) \leq p(t) \psi(v(t)), \quad t \in J .
$$

This implies that for each $t \in J$,

$$
\int_{v(0)}^{v(t)} \frac{d u}{\psi(u)} \leq \int_{0}^{b} p(s) d s<+\infty .
$$

This inequality, together with hypothesis (H7), implies that there exists a constant $d$ such that $v(t) \leq d, t \in J$, and hence $\|y\|_{\infty} \leq d$, where $d$ depends only on the functions $p$ and $\psi$. This shows that $\mathscr{E}\left(N_{1}\right)$ is bounded. As a consequence of Schaefer's theorem [12], we deduce that $N_{1}$ has a fixed point $y$ which is a solution to problem (2.24). Then $y$ is a solution to problem (1.1).

\section{References}

[1] M. Benchohra and S. K. Ntouyas, Existence of mild solutions of semilinear evolution inclusions with nonlocal conditions, Georgian Math. J. 7 (2000), no. 2, 221-230.

[2] A. Bielecki, Une remarque sur la méthode de Banach-Cacciopoli-Tikhonov dans la théorie des équations différentielles ordinaires, Bull. Acad. Polon. Sci. Cl. III. 4 (1956), 261-264 (French).

[3] A. Bressan and G. Colombo, Extensions and selections of maps with decomposable values, Studia Math. 90 (1988), no. 1, 69-86.

[4] L. Byszewski, Theorems about the existence and uniqueness of solutions of a semilinear evolution nonlocal Cauchy problem, J. Math. Anal. Appl. 162 (1991), no. 2, 494-505.

[5] _ Existence and uniqueness of a classical solution to a functional-differential abstract nonlocal Cauchy problem, J. Appl. Math. Stochastic Anal. 12 (1999), no. 1, 91-97.

[6] C. Castaing and M. Valadier, Convex Analysis and Measurable Multifunctions, Lecture Notes in Mathematics, vol. 580, Springer-Verlag, New York, 1977.

[7] H. Covitz and S. B. Nadler Jr., Multivalued contraction mappings in generalized metric spaces, Israel J. Math. 8 (1970), 5-11.

[8] K. Deimling, Multivalued Differential Equations, de Gruyter Series in Nonlinear Analysis and Applications, vol. 1, Walter de Gruyter, Berlin, 1992.

[9] M. Frigon and A. Granas, Théorèmes d'existence pour des inclusions différentielles sans convexité [Existence theorems for differential inclusions without convexity], C. R. Acad. Sci. Paris Sér. I Math. 310 (1990), no. 12, 819-822 (French). 


\section{On a nonlocal Cauchy problem for differential inclusions}

[10] L. Górniewicz, Topological Fixed Point Theory of Multivalued Mappings, Mathematics and Its Applications, vol. 495, Kluwer Academic Publishers, Dordrecht, 1999.

[11] Sh. Hu and N. S. Papageorgiou, Handbook of Multivalued Analysis. Vol. I. Theory, Mathematics and Its Applications, vol. 419, Kluwer Academic Publishers, Dordrecht, 1997.

[12] D. R. Smart, Fixed Point Theorems, Cambridge Tracts in Mathematics, no. 66, Cambridge University Press, London, 1974.

[13] A. A. Tolstonogov, Differential Inclusions in a Banach Space, Mathematics and Its Applications, vol. 524, Kluwer Academic Publishers, Dordrecht, 2000.

E. Gatsori: Department of Mathematics, University of Ioannina, 45110 Ioannina, Greece E-mail address: me00416@cc.uoi.gr

S. K. Ntouyas: Department of Mathematics, University of Ioannina, 45110 Ioannina, Greece E-mail address: sntouyas@cc.uoi.gr

Y. G. Sficas: Department of Mathematics, University of Ioannina, 45110 Ioannina, Greece E-mail address: ysficas@cc.uoi.gr 


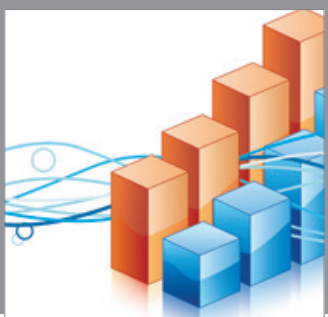

Advances in

Operations Research

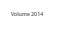

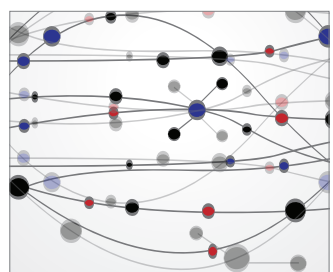

\section{The Scientific} World Journal
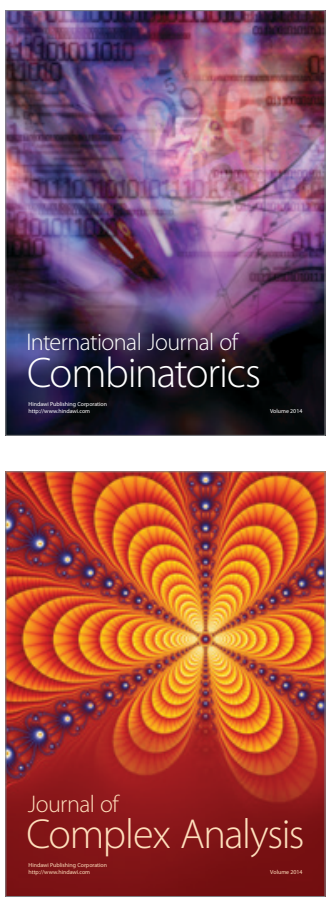

International Journal of

Mathematics and

Mathematical

Sciences
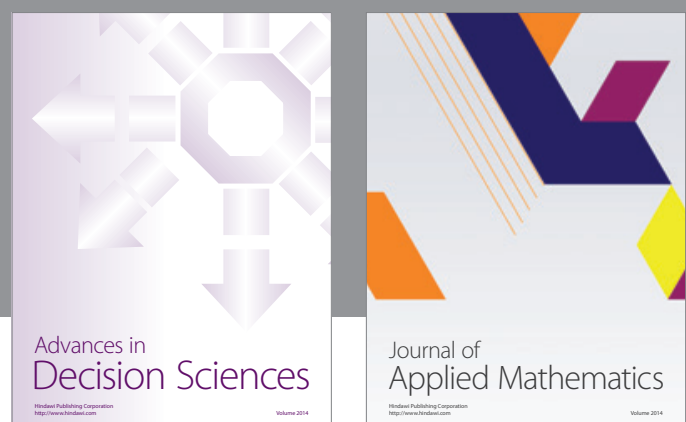

Journal of

Applied Mathematics
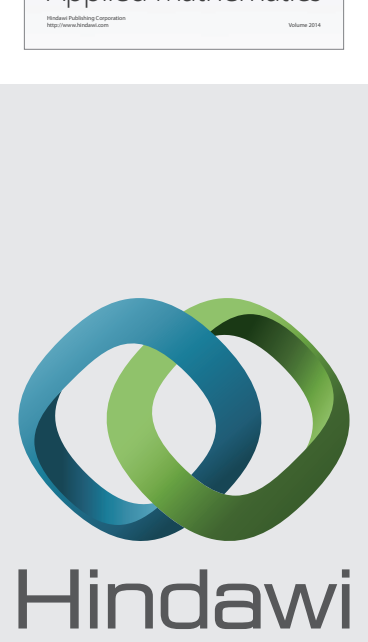

Submit your manuscripts at http://www.hindawi.com
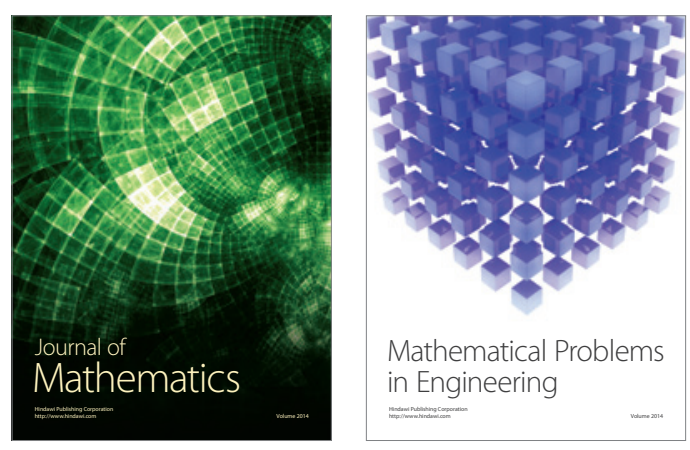

Mathematical Problems in Engineering
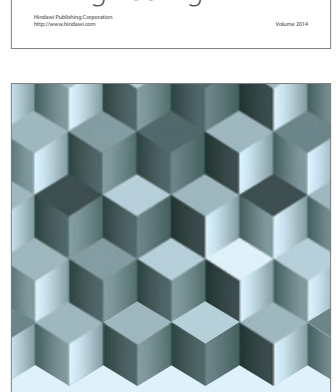

Journal of

Function Spaces
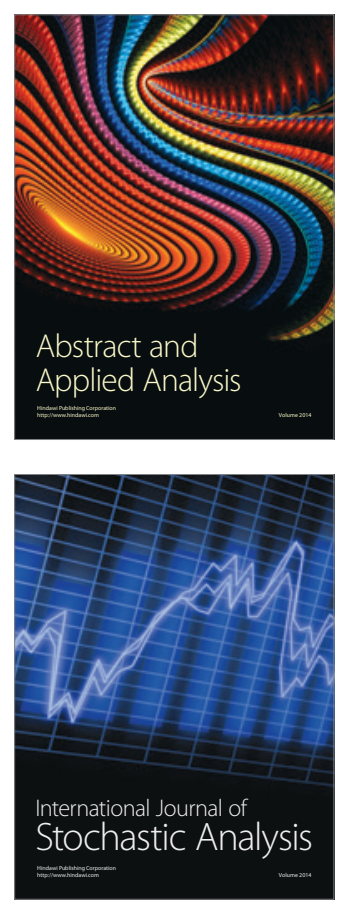

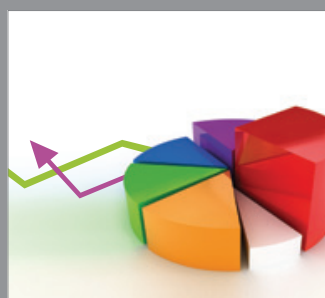

ournal of

Probability and Statistics

Promensencen
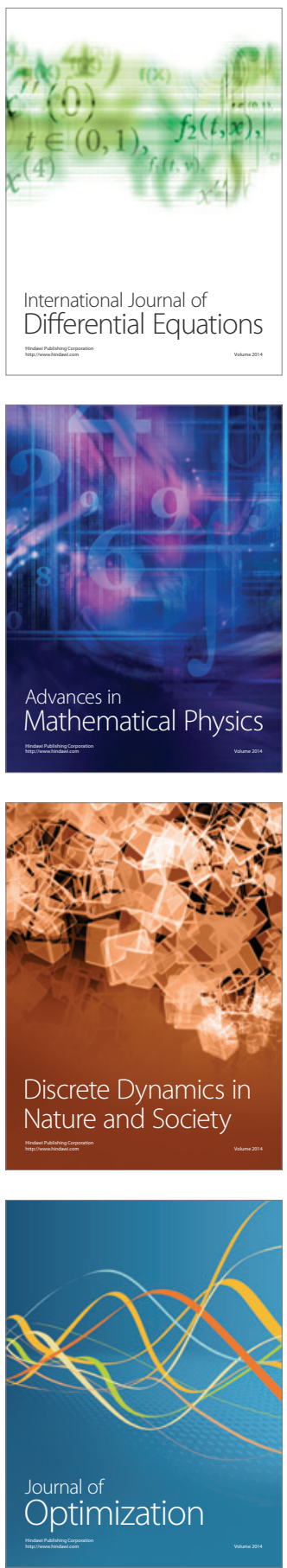